\title{
Photophysics of Isolated Rose Bengal Anions
}

Mark H. Stockett, ${ }^{\dagger}$ Christina Kjær, ${ }^{\ddagger}$ Steven Daly, ${ }^{\pi}, \#$ Evan J. Bieske, ${ }^{\S}$ Jan R. R. Verlet, $\|$ Steen Brøndsted Nielsen, ${ }^{\ddagger}$ and James N. Bull ${ }^{*, \perp}$

$\dagger$ Department of Physics, Stockholm University, SE-10691 Stockholm, Sweden $\ddagger$ Department of Physics and Astronomy, Aarhus University, Aarhus 8000, Denmark

\Univ Lyon, Université Claude Bernard Lyon 1, CNRS, Institut Lumiére Matiére UMR 5306, F-69100, Villeurbanne, France

$\S$ School of Chemistry, University of Melbourne, Parkville, VIC 3010, Australia ||Department of Chemistry, Durham University, Durham, DH1 3LE, United Kingdom $\perp$ School of Chemistry, Norwich Research Park, University of East Anglia, Norwich NR 4 7TJ, United Kingdom

\#Current address: MSVision, Spectrometry Vision B.V., Televisieweg 40, 1322 AM Almere, The Netherlands

E-mail: james.bull@uea.ac.uk 


\begin{abstract}
Dye molecules based on the xanthene moiety are widely used as fluorescent probes in bioimaging and technological applications due to their large absorption cross-section for visible light and high fluorescence quantum yield. These applications require a clear understanding of the dye's inherent photophysics and the effect of a condensed-phase environment. Here, the gas-phase photophysics of Rose Bengal doubly deprotonated dianion $[\mathrm{RB}-2 \mathrm{H}]^{2-}$, deprotonated monoanion $[\mathrm{RB}-\mathrm{H}]^{-}$and doubly deprotonated radical anion $[\mathrm{RB}-2 \mathrm{H}]^{\bullet-}$, are investigated using photodetachment, photoelectron, tandem ion mobility spectrometry (IMS) coupled with laser excitation, and dispersed fluorescence action spectroscopies. For $[\mathrm{RB}-2 \mathrm{H}]^{2-}$, photodetachment action spectroscopy reveals a clear band in the visible $(450-580 \mathrm{~nm})$ with vibronic structure. Electron affinity and repulsive Coulomb barrier ( $\mathrm{RCB}$ ) properties of the dianion are characterized using frequency-resolved photoelectron spectroscopy, revealing a decreased RCB compared with fluorescein dianions due to electron delocalization over halogen atoms. Monoanions $[\mathrm{RB}-\mathrm{H}]^{-}$and $[\mathrm{RB}-2 \mathrm{H}]^{\bullet-}$ differ in nominal mass by $1 \mathrm{Da}$, but are difficult to study using action spectroscopies that isolate target ions using low-resolution mass spectrometry. This work shows that the two monoanions are readily distinguished and probed using the IMS-photo-IMS and photo-IMSphoto-IMS strategies, providing distinct but overlapping photodissociation action spectra in the visible spectral range. Gas-phase fluorescence was not detected from $[\mathrm{RB}-2 \mathrm{H}]^{2-}$ due to rapid electron ejection. However, both $[\mathrm{RB}-\mathrm{H}]^{-}$and $[\mathrm{RB}-2 \mathrm{H}]^{\bullet-}$ show weak fluorescence signal. The $[\mathrm{RB}-\mathrm{H}]^{-}$action spectra show a large Stokes shift of $\approx 1,700 \mathrm{~cm}^{-1}$, while the $[\mathrm{RB}-2 \mathrm{H}]^{\bullet-}$ action spectra show no appreciable Stokes shift. This difference is explained by considering geometries of the ground and fluorescing states.
\end{abstract}




\section{Introduction}

The xanthene moiety is a conjugated tricyclic ring that serves as the functional base for many synthetic dye molecules, including the rhodamines and fluorones. ${ }^{1,2}$ Dyes containing the xanthene unit are commonly employed as fluorescent probes in biophysical and technological applications due to their large absorption crosssections and high fluorescence quantum yield in most condensed phase environments. ${ }^{2-4}$ For example, fluorescent probes based on fluorone dyes are widely used as tracers in angiography, ${ }^{5}$ cellular imaging agents and stains in histology, ${ }^{6}$ diagnostic probes for detecting trace amounts of peptides, ${ }^{7}$ and as donors and acceptors in FRET (Förster Resonance Energy Transfer) complexes used to elucidate molecular structure. ${ }^{8,9}$

Rapid advances in the bioimaging and biotagging fields over the last decade have prompted a range of experimental and theoretical investigations on the fundamental photophysical properties of xanthene-containing dye molecules. ${ }^{10-16}$ In this context, the gas phase provides a unique environment to explore their inherent photophysics due to the absence of intermolecular interactions and straight-forward comparisons of experimental results with theory. ${ }^{17,18}$

Gas-phase action spectroscopy techniques applied to anion (as opposed to cation) xanthene-based dye molecules include electrospray ionization coupled with photodissociation action spectroscopy to infer gas-phase absorption spectra, ${ }^{19-22}$ ion traps coupled with laser excitation and fluorescence detection to record emission spectra, ${ }^{21-24}$ and photoelectron spectroscopy in the frequency and time domains. ${ }^{25,26}$ The emerging technique of IMS coupled with laser excitation has been used to elucidate the isomeric forms and provide isomer-specific action spectra for several cation dye molecules. ${ }^{16,27-29}$ While each of these techniques provides insight into the photophysical properties of the target ion(s), rarely have the techniques been deployed in concert.

The most thoroughly studied xanthene-based anion dye in the gas phase is fluorescein, which is usually generated as the singly deprotonated anion, $[\mathrm{F}-\mathrm{H}]^{-}$, and the doubly deprotonated anion, $[\mathrm{F}-2 \mathrm{H}]^{2-}$, using electrospray ionization. Photodissociation action spectroscopy has been used to infer absorption spectra for $[\mathrm{F}-\mathrm{H}]^{-}$and a range of halogenated derivatives. ${ }^{20}$ Timeresolved photodetachment action spectroscopy on [F$\mathrm{H}]^{-}$has been used to characterize rotational and vibrational wavepacket dynamics following excitation of the $\mathrm{S}_{1}\left(\pi \pi^{*}\right)$ state. ${ }^{26}$ Photon counting experiments for $[\mathrm{F}-\mathrm{H}]^{-}$in the gas phase suggest that the $\mathrm{S}_{1}\left(\pi \pi^{*}\right)$ state pumped at $510 \mathrm{~nm}$ survives for $\approx 5 \mathrm{~ns} .{ }^{22}$ For $[\mathrm{F}-\mathrm{H}]^{-}$, the long gas-phase $\mathrm{S}_{1}\left(\pi \pi^{*}\right)$ state lifetime and substantial difference between ground and excited state geometries leads to good fluorescence signal with the largest known Stokes shift for a gas-phase dye $\left(\approx 2,000 \mathrm{~cm}^{-1}\right) .{ }^{23,24}$

The $[\mathrm{F}-2 \mathrm{H}]^{2-}$ species (doubly deprotonated dianion) has different excited state dynamics than the singly deprotonated monoanion due in part to the presence of a repulsive Coulomb barrier ( $\mathrm{RCB}$ ) to electron detachment. ${ }^{30,31}$ Briefly, an RCB is a property of multiply charged anions resulting from the combination of shortrange attraction forces and long-range Coulomb repulsion forces, leading to a potential energy barrier for electron ejection. Attempts at measuring fluorescence from $[\mathrm{F}-2 \mathrm{H}]^{2-}$ were unsuccessful presumably because autodetachment is more rapid than fluorescence. ${ }^{20}$ Subsequent time-resolved measurements on the $\mathrm{S}_{1}\left(\pi \pi^{*}\right)$ state of gas-phase $[\mathrm{F}-2 \mathrm{H}]^{2-}$ found an excited state lifetime of $0.9 \pm 0.2 \mathrm{ps}$, which was independent of excitation energy. ${ }^{25}$ The principal decay pathway of the photoexcited anion is electron loss by autodetachment.

This paper considers the gas-phase photophysics of deprotonated forms of Rose Bengal (RB), which is a halogenated derivative of fluorescein. Like fluorescein, RB possesses a large $\mathrm{S}_{1}\left(\pi \pi^{*}\right) \leftarrow \mathrm{S}_{0}$ absorption crosssection for visible light and high luminescence quantum yield in most solvents. ${ }^{2}$ In polar solvents, RB exists as the doubly deprotonated anion, $[\mathrm{RB}-2 \mathrm{H}]^{2-}$, as shown in Figure 1. Electrospray ionization of RB from a protic solvent generates the deprotonated monoanion (closed shell), [RB-H $]^{-}$, potentially in both phenoxide and carboxylate deprotomeric forms, and also the doubly deprotonated radical monoanion (open shell), $[\mathrm{RB}-2 \mathrm{H}]^{\bullet-}$. This study addresses several questions: (i) Does each gas-phase form have distinct photophysics? (ii) What are the RCB properties of the dianion and how do these compare with those for $[\mathrm{F}-2 \mathrm{H}]^{2-}$ ? (iii) Is $[\mathrm{RB}-\mathrm{H}]^{-}$generated from methanol (protic solvent) as a single deprotomer using electrospray ionization? (iv) Is it possible to isolate $[\mathrm{RB}-\mathrm{H}]^{-}$from $[\mathrm{RB}-2 \mathrm{H}]^{\bullet-}$, considering that they differ in nominal mass by $1 \mathrm{Da}$ but have broad isotope distributions, and record action spectra for each? (v) Do any of the anions fluoresce in the gas phase and what is the magnitude of their Stokes shift? These ques-

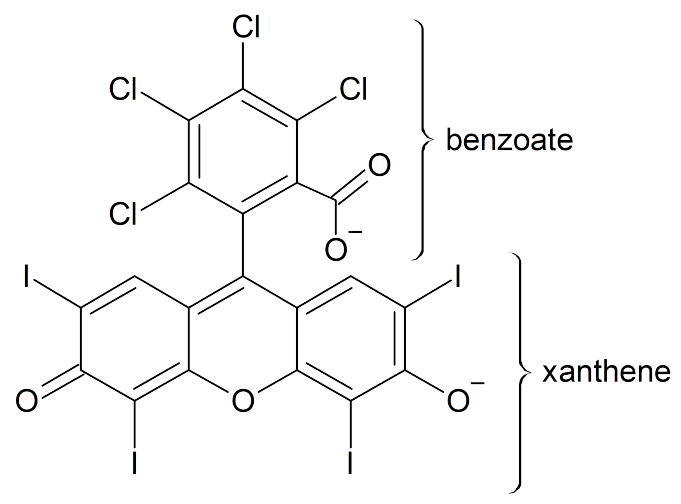

Figure 1: Doubly deprotonated Rose Bengal (4,5,6,7tetrachloro- $2^{\prime}, 4^{\prime}, 5^{\prime}, 7^{\prime}$-tetraiodofluorescein), $[\mathrm{RB}-2 \mathrm{H}]^{2-}$, showing the xanthene and benzoate units. For [RB$\mathrm{H}]^{-}$, the xanthene unit is deprotonated and the benzoate unit exists as a benzoic acid. Note, this molecule is often referred to as Rose Bengal B (purchased as the $>99 \%$ purity disodium salt, Sigma-Aldrich), and differs from Rose Bengal A (lactone functional group). 
tions are addressed by performing complementary action spectroscopy measurements on four purpose-built instruments.

\section{Methods}

\section{Photodetachment spectroscopy}

Photodetachment action spectroscopy of $[\mathrm{RB}-2 \mathrm{H}]^{2-}$, which involved monitoring formation of $[\mathrm{RB}-2 \mathrm{H}]^{\bullet-}$, was performed using the Sep1 accelerator mass spectrometer at Aarhus University. ${ }^{32,33}$ Electrosprayed anions were accumulated in an octupole ion trap that was emptied every $25 \mathrm{~ms}(40 \mathrm{~Hz}$ repetition rate). The ion bunches were accelerated to a kinetic energy of $\approx 50 \mathrm{keV}$ and mass selected using a bending magnet. A nanosecond-pulsed laser system (EKSPLA NT342A, $20 \mathrm{~Hz}$, unfocused) excited every second ion bunch midway along a $2.5 \mathrm{~m}$ linear flight region (pressure $10^{-6}$ Torr). Daughter ions were separated using an electrostatic energy analyser situated after the laser-ion interaction region and detected with a channeltron. For a given wavelength, the difference in the number of counts between the 'lighton' and 'light-off' injections provided the photoinduced signal. Photoinduced events occurring up to $10 \mu \mathrm{s}$ after excitation are observed, i.e. limited by ion traversal time through the flight region. The light fluence was adjusted such that photodetachment was the predominant channel (see Supporting Information).

\section{Photoelectron spectroscopy}

Photoelectron spectroscopy measurements using the frequency- and angle-resolved photoelectron imaging strategy $^{34,35}$ were performed at Durham University using an instrument that combines electrospray ionization, ion trapping, time-of-flight mass separation and velocity-map imaging detection. ${ }^{36,37}$ Electrosprayed ions from methanol solution were accumulated in a radio frequency ring-electrode ion trap. The trapped anions were unloaded $(10 \mathrm{~Hz}$ repetition rate) into a colinear time-of-flight optics assembly that accelerated the ions along a $1.3 \mathrm{~m}$ flight region toward a continuous-mode penetrating field velocity-map imaging assembly. ${ }^{37}$ Light from a nanosecond-pulsed laser system (Continuum Horizon OPO pumped by a Continuum Surelite II Nd:YAG laser, $10 \mathrm{~Hz}$, unfocused, $<0.5 \mathrm{~mJ} \mathrm{~cm}^{-2}$ pulse $^{-1}$ ) was timed to interact with a mass-selected ion packet at the centre of the velocity map imaging stack. Ejected electrons were velocitymapped onto a dual (chevron) multichannel plate detector, followed by a P43 phosphor screen, which was monitored with a charge-coupled device camera. Velocity map images were accumulated with a $500 \mathrm{~ns}$ microchannel plate gate, which is required for velocitymap imaging. The velocity mapping resolution was $\frac{\Delta E}{E} \approx 5 \%$, and the electron kinetic energy (eKE) scale was calibrated from the spectrum of $\mathrm{I}^{-}$. Velocitymap image reconstructions used antialiasing and polar onion-peeling algorithms, ${ }^{38}$ providing the photoelectron spectra and associated photoelectron angular distributions quantified by the $\beta_{2}$ anisotropy parameter. ${ }^{39}$

\section{Tandem ion mobility spectrometry}

Photodetachment and photodissociation of $[\mathrm{RB}-2 \mathrm{H}]^{2-}$, $[\mathrm{RB}-\mathrm{H}]^{-}$, and $[\mathrm{RB}-2 \mathrm{H}]^{\bullet-}$ was investigated in a custom tandem ion mobility spectrometer (IMS-IMS) and quadrupole mass filter (QMF) apparatus at The University of Melbourne. ${ }^{27,40}$ Target anions were produced through electrospray ionization of $\mathrm{a} \approx 10 \mu \mathrm{mol} \mathrm{L}^{-1}$ solution of Rose Bengal dissolved in dry acetonitrile or methanol (voltage $-3 \mathrm{kV}$, flow rate $\approx 10 \mu \mathrm{L} \mathrm{min}^{-1}$ ). Electrosprayed ions were transferred via a heated capillary into a RF ion funnel (IF1), which radially gathered and confined the ions. An ion gate situated at the end of IF1 injected $\approx 100 \mu$ s packets of ions at $40 \mathrm{~Hz}$ into the first IMS drift region (IMS1) where they were propelled by an electric field ( $44 \mathrm{~V} \mathrm{~cm}^{-1}$ ) through $\mathrm{N}_{2}+\approx 1 \% \mathrm{SF}_{6}$ buffer gas (dianion) or pure $\mathrm{N}_{2}$ (monoanions) at a pressure of $\approx 6$ Torr. The different isomeric forms are separated spatially and temporally because of differences in their collision cross-sections. ${ }^{41}$ After ions traversed both drift regions (IMS1 + IMS2), a second ion funnel (IF2) collected the ions and introduced them into a differentially pumped octupole ion guide and QMF that mass-selected the ions before they reached the ion detector. The detector was connected to a multichannel scaler that produced a histogram of ion counts against arrival time, $t$, corresponding to an arrival time distribution (ATD). In all presented ATDs, $t=0$ corresponds to the opening of IG1. The mobility resolution, $t / \Delta t$, for a singly-charged anion is typically 80-90.

For the IMS-photo-IMS measurements, packets of ions with similar collision cross-sections were selected using a Bradbury-Nielsen ion gate after IMS1 $(\approx 100 \mu \mathrm{s}$ opening time). Immediately after this, the mobilityselected ions were excited with a pulse of light from an optical parametric oscillator (OPO, EKSPLA NT342B, unfocused). The OPO was operated at $20 \mathrm{~Hz}$, which was half the rate of ion injection, allowing accumulation of light-on and light-off ATDs. The difference between the light-on and light-off ATDs reflected the photoresponse (photoaction ATD). Action spectra were derived by integrating the photoaction ATD signal and normalizing with respect to light pulse fluence and total laser-off signal at each wavelength. The photodetachment measurements for $[\mathrm{RB}-2 \mathrm{H}]^{2-}$ were performed with a light fluence of $<0.4 \mathrm{~mJ} \mathrm{~cm}^{-2}$ pulse $^{-1}$. The photodissociation measurements for $[\mathrm{RB}-\mathrm{H}]^{-}$and $[\mathrm{RB}-2 \mathrm{H}]^{\bullet-}$ were performed with a light pulse fluence of $\approx 2 \mathrm{~mJ} \mathrm{~cm}{ }^{-2}$ pulse $^{-1}$.

Action spectroscopy on $[\mathrm{RB}-2 \mathrm{H}]^{\bullet-}$ was performed using the photo-IMS-photo-IMS strategy. The [RB$2 \mathrm{H}]^{\bullet-}$ ions were photogeneration by intercepting electrosprayed $[\mathrm{RB}-2 \mathrm{H}]^{2-}$ ions with a pulse of $532 \mathrm{~nm}$ light (Quantel Big Sky Nd:YAG laser, $\approx 40 \mathrm{~mJ} \mathrm{~cm}^{-2}$ pulse $^{-1}$, $40 \mathrm{~Hz}$, unfocused) immediately after the ions were injected into the drift region. ${ }^{42,43}$ This wavelength is close 
to the maximum in the $[\mathrm{RB}-2 \mathrm{H}]^{2-}$ photodetachment action spectrum, ensuring efficient production of [RB$2 \mathrm{H}]^{\bullet-}$.

\section{Gas-phase dispersed fluorescence}

Gas-phase dispersed fluorescence spectroscopy was performed using the LUminescence iNstrument in Aarhus (LUNA) apparatus. ${ }^{44,45}$ Briefly, electrosprayed ions from methanol solution were accumulated in an octupole ion trap and transferred into a cylindrical Paul trap $(20 \mathrm{~Hz}$ repetition rate) where they became thermalized with helium buffer gas. The electrostatic fields on the Paul trap were tuned to trap the anion of interest. Trapped ions were irradiated with $533 \mathrm{~nm}$ light from an EKSPLA NT342A OPO $(20 \mathrm{~Hz}, \approx 5 \mathrm{~ns}$ pulse duration, focused, $\approx 2 \mathrm{~mW}$ ). Emitted photons were collected using a lens mounted on one trap endcap ( $\mathrm{f}=15 \mathrm{~mm}$, $\mathrm{NA}=0.57$ ), focused ( $100 \mathrm{~mm}$ achromatic lens) into the entrance of a grating spectrograph (Andor 303i), and detected using a CCD camera (Andor Newton). A $533 \mathrm{~nm}$ notch filter (Thorlabs NF533-17) situated between the collection and focussing lenses prevented scattered laser light reaching the detector. Emission spectra were acquired over 100 repeat cycles of 'ions-on' and 'ions-off' spectral acquisitions. The final emission spectrum is the difference between the total ions-on and ions-off spectra.

\section{Solution absorption spectra}

Solution absorption spectra of $[\mathrm{RB}-2 \mathrm{H}]^{2-}$ were recorded with Varian Cary Bio spectrophotometer using a quartz cuvette with sample concentrations adjusted to achieve an absorbance of less than 0.1 at the peak absorption wavelength.

\section{Computational}

Electronic structure calculations of geometries, vibrational frequencies, and vertical excitation energies for the isolated anions were performed using the Gaussian 16.B01, ${ }^{46}$ ORCA 4.2.1, ${ }^{47}$ and MRCC (February 2020 release) software packages. ${ }^{48}$ Geometry optimizations and vibrational frequency calculations were performed at the $\omega \mathrm{B} 97 \mathrm{X}-\mathrm{D} / \mathrm{def} 2-\mathrm{SVPD}$ and LC- $\omega \mathrm{HPBE} / \mathrm{def} 2-$ SVPD levels of theory. ${ }^{49-52}$ The def2-SVPD basis set includes an effective core potential for iodine atoms. ${ }^{53}$ Adiabatic detachment energy (ADE) and vertical detachment energy (VDE) parameters were computed at the DLPNO-CCSD(T)/def2-SVPD level of theory assuming the $\omega \mathrm{B} 97 \mathrm{X}-\mathrm{D} /$ def2-SVPD geometries. FranckCondon-Herzberg-Teller (FCHT) simulation of absorption spectrum for $[\mathrm{RB}-2 \mathrm{H}]^{2-}$ was performed at the $\omega \mathrm{B} 97 \mathrm{X}-\mathrm{D} /$ def2-SVPD level of theory assuming the temperature of $300 \mathrm{~K}$ (i.e. including hot band contributions) using the FCHT method as implemented in Gaussian 16.B01. ${ }^{54}$ Vertical excitation (VE) wavelength values for the $\mathrm{S}_{1}\left(\pi \pi^{*}\right) \leftarrow \mathrm{S}_{0}$ transition were computed at the df-CC2/def2-SVPD, df-ADC(2)/def2SVPD, and DLPNO-STEOM-CCSD/def2-SVPD levels of theory. ${ }^{55-57}$ Selected VE wavelengths were computed with the def2-TZVPD (triplet- $\zeta$ ) basis set to assess the performance of the def2-SVPD (double- $\zeta$ ) basis set.

Collision cross-sections were calculated using MOBCAL with the trajectory method parametrised for $\mathrm{N}_{2}$ buffer gas. ${ }^{58,59}$ Input charge distributions were computed at the $\omega \mathrm{B} 97 \mathrm{X}-\mathrm{D} /$ def2-SVPD level of theory with the Merz-Singh-Kollman scheme constrained to reproduce the electric dipole moment. ${ }^{60}$ Sufficient trajectories were computed to give standard deviations of $\pm 1 \AA^{2}$ for the calculated values. Note that the present version of MOBCAL was parametrized for cations and its performance for anions has not been benchmarked.

\section{Results and Discussion}

\section{[RB-2H $^{2-}$ (dianion)}

\section{Photodetachment action spectroscopy}

Photodetachment action spectra for $[\mathrm{RB}-2 \mathrm{H}]^{2-}$ are shown in Figure 2a. In the Sep1 measurement, photodetachment was monitored through formation of [RB$\mathrm{H}]^{\bullet-}$. In the IMS measurement, photodetachment was monitored through formation of $\mathrm{SF}_{6}^{-}$(this spectrum is essentially identical to the one obtained by monitoring $[\mathrm{RB}-\mathrm{H}]^{\bullet-}$ in the same experiment - see Supporting Information). In all cases, the wavelength of maximum response is $538 \pm 5 \mathrm{~nm}$, with shoulder peaks at $506 \pm 5$ and $465 \pm 5 \mathrm{~nm}$. A FCHT simulation of the absorption spectrum at $T=300 \mathrm{~K}$ is shown in Figure $2 \mathrm{~b}$, which has been translated (vertical excitation wavelength predicted at $434 \mathrm{~nm}$ ) so that the band maximum agrees with the main peak in panel a. The simulation predicts that the main peak in the action spectrum is due to an extended progression of the $13 \mathrm{~cm}^{-1}$ mode (torsion of the ring systems) with substantial contributions from combination modes and hot bands. The shoulder peaks observed at $506 \pm 5$ and $465 \pm 5 \mathrm{~nm}$ are predominantly from a progression of the $1550 \mathrm{~cm}^{-1}$ mode (xanthene symmetric stretch). Calculated vibrational frequencies are tabulated in the Supporting Information.

The absorption spectrum of $[\mathrm{RB}-2 \mathrm{H}]^{2-}$ in acetonitrile (non-protic solvent) is shown in Figure 2c and has a maximum response at $558 \pm 1 \mathrm{~nm}$. The spectral profile closely resembles the gas-phase action spectra, although it is red shifted by $\approx 20 \mathrm{~nm}$. The red shift in solution will be in part due to a change in charge distribution and also an increased polarizability in the excited state (calculated gas-phase isotropic polarizability, $\alpha_{i s o}=82.1 \AA^{3}$ ), leading to stronger interactions with solvent molecules compared with the $\mathrm{S}_{0}$ state $\left(\alpha_{\text {iso }}=80.5 \AA^{3}\right) .{ }^{23,24}$

Calculated vertical excitation (VE) wavelengths for $[\mathrm{RB}-2 \mathrm{H}]^{2-}$ are given in Table 1. Both $\omega$ B97X-D $(424 \mathrm{~nm})$ and LC- $\omega$ HPBE $(420 \mathrm{~nm})$ values are in poor agreement with the experimental value, presumably due 

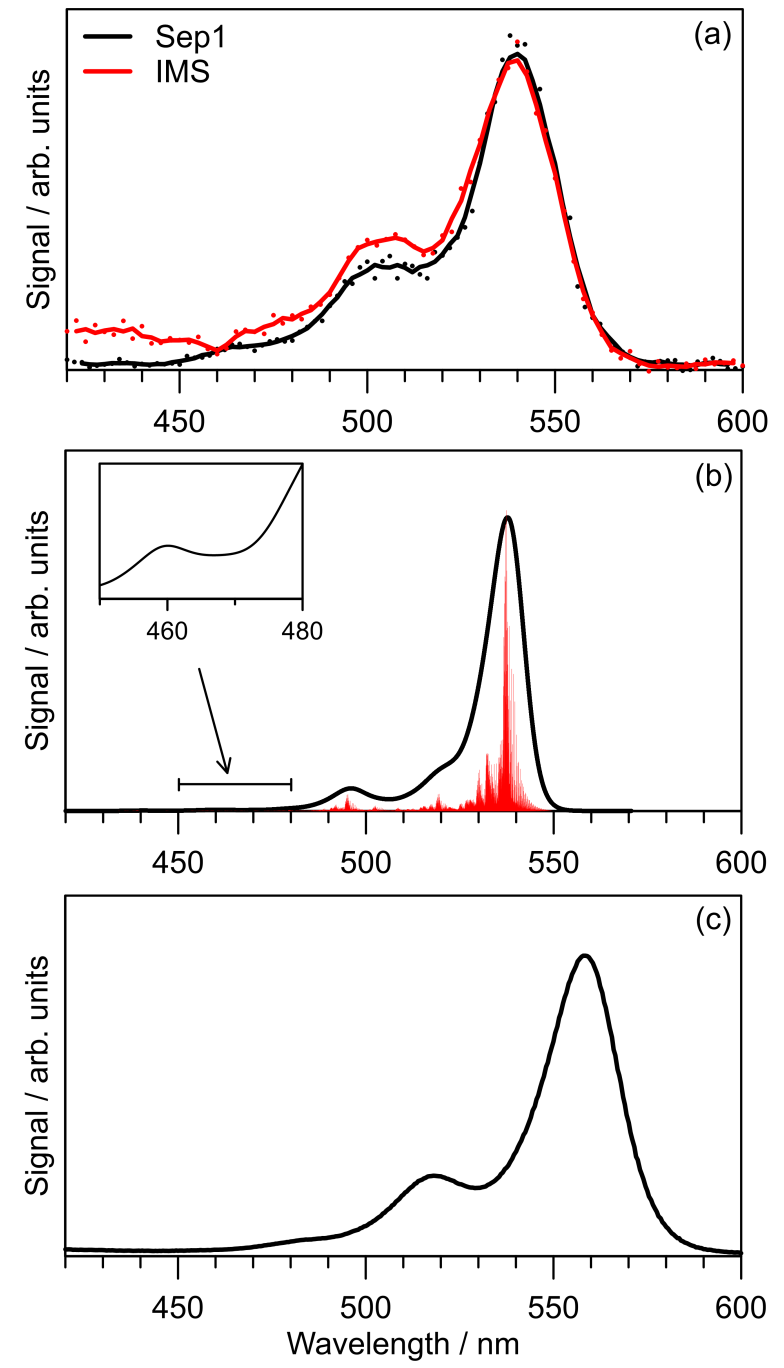

Figure 2: $[\mathrm{RB}-2 \mathrm{H}]^{2-}$ spectroscopy: (a) photodetachment action spectrum recorded by monitoring formation of $[\mathrm{RB}-\mathrm{H}]^{\bullet-}$ using the Sep1 instrument and by monitoring $\mathrm{SF}_{6}^{-}$formation using the tandem IMS instrument, (b) FCHT simulation of the $\mathrm{S}_{1}\left(\pi \pi^{*}\right) \leftarrow \mathrm{S}_{0}$ absorption spectrum, (c) absorption spectrum in dry acetonitrile.

to an inadequate description of the transition's chargetransfer character. Similar conclusions were reached in a systematic computational study on other xanthenecontaining dyes. ${ }^{61}$ The df-CC2 $(501 \mathrm{~nm})$ and STEOMDLPNO-CCSD $(568 \mathrm{~nm})$ values are in better agreement with the experimental value. Increasing the basis set quality to triple- $\zeta$ gives little improvement to the df$\mathrm{CC} 2$ value. We suspect that improving description of doubles (and triples) excitations in the electron correlation space is needed to achieve satisfactory agreement between theory and experiment.

\section{Photoelectron Spectroscopy and the RCB}

Electron detachment processes that ensue after excitation of the $\mathrm{S}_{1}\left(\pi \pi^{*}\right) \leftarrow \mathrm{S}_{0}$ band of $[\mathrm{RB}-2 \mathrm{H}]^{2-}$ were characterized using the frequency- and angle-resolved pho- toelectron spectroscopy strategy. ${ }^{34,35}$ Briefly, the technique involves recording photoelecton velocity-map images for a series of wavelengths across the absorption band (14 in this case). The velocity-map images contain details of excited state dynamics embodied in the radial and angular dimensions. The photoelectron angular distributions are quantified in terms of $\beta_{2}$ values, ${ }^{39}$ which range from -1 to 2 , corresponding to electron ejection perpendicular and parallel to the laser polarization, respectively. Example photoelectron spectra recorded at 580, 520 and $400 \mathrm{~nm}$ are shown in Figure $3 \mathrm{a}$. The frequency-resolved photoelectron spectrum is shown in Figure $3 \mathrm{~b}$ where the horizontal abscissa is electron kinetic energy (eKE) and the vertical abscissa is photon energy or wavelength. Each photoelectron spectrum making up the frequency-resolved spectrum has been normalized to its maximum intensity to accentuate spectral features. The total photoelectron yield, taken from the Sep1 photodetachment spectrum from Figure $2 \mathrm{a}$, is shown alongside the frequency-resolved spec-

Table 1: $\mathrm{S}_{1}\left(\pi \pi^{*}\right) \leftarrow \mathrm{S}_{0}$ vertical excitation (VE) wavelengths in $\mathrm{nm}$, and vertical (VDE) and adiabatic (ADE) electron detachment energies in $\mathrm{eV} .\left(\mathrm{O}^{-}\right)$and $\left(\mathrm{COO}^{-}\right)$ indicate phenoxide and carboxylate deprotomers of $[\mathrm{RB}-\mathrm{H}]^{-}$, respectively, and are further discussed in the ion mobility spectrometry section. Note, df-CC2 and df-ADC(2) calculations using MRCC are not possible for open-shell species. ${ }^{a}$ STEOM is an abbreviation for STEOM-DLPNO-CCSD. ${ }^{b}$ Value in parentheses uses the def2-TZVPD basis set.

\begin{tabular}{|c|c|c|c|}
\hline$[\mathbf{R B}-2 \mathrm{H}]^{2-}$ & $\mathrm{S}_{1} \mathrm{VE}$ & VDE & $\mathrm{ADE}$ \\
\hline$\omega \mathrm{B} 97 \mathrm{X}-\mathrm{D}$ & 434 & - & - \\
\hline LC- $\omega \mathrm{HPBE}$ & 420 & - & - \\
\hline $\mathrm{df}-\mathrm{CC} 2$ & $501(505)^{b}$ & - & - \\
\hline df-ADC (2) & 570 & - & - \\
\hline $\mathrm{STEOM}^{a}$ & 568 & - & - \\
\hline DLPNO-CCSD(T) & - & 1.31 & 1.22 \\
\hline Experimental & $538 \pm 5$ & $1.3 \pm 0.1$ & $1.1 \pm 0.1$ \\
\hline$[\mathrm{RB}-\mathrm{H}]^{-}\left(\mathrm{O}^{-}\right)$ & $\mathrm{S}_{1} \mathrm{VE}$ & VDE & $\mathrm{ADE}$ \\
\hline$\omega \mathrm{B} 97 \mathrm{X}-\mathrm{D}$ & 454 & - & - \\
\hline LC- $\omega \mathrm{HPBE}$ & 437 & - & - \\
\hline $\mathrm{df}-\mathrm{CC} 2$ & $508(514)^{b}$ & - & - \\
\hline df-ADC $(2)$ & 578 & - & - \\
\hline DLPNO-CCSD(T) & - & 4.17 & 4.14 \\
\hline Experimental & $545 \pm 10$ & - & - \\
\hline$[\mathrm{RB}-\mathrm{H}]^{-}\left(\mathrm{COO}^{-}\right)$ & $\mathrm{S}_{1} \mathrm{VE}$ & VDE & $\mathrm{ADE}$ \\
\hline$\omega \mathrm{B} 97 \mathrm{X}-\mathrm{D}$ & 415 & - & - \\
\hline LC- $\omega \mathrm{HPBE}$ & 364 & - & - \\
\hline df-CC2 & 497 & - & - \\
\hline df-ADC(2) & 547 & - & - \\
\hline DLPNO-CCSD(T) & - & - & 3.50 \\
\hline [RB-2H] ${ }^{--}$ & $\mathrm{S}_{1} \mathrm{VE}$ & VDE & $\mathrm{ADE}$ \\
\hline$\omega \mathrm{B} 97 \mathrm{X}-\mathrm{D}$ & 414 & - & - \\
\hline LC- $\omega \mathrm{HPBE}$ & 521 & - & - \\
\hline DLPNO-CCSD(T) & - & - & 3.16 \\
\hline Experimental & $563 \pm 5$ & - & - \\
\hline
\end{tabular}



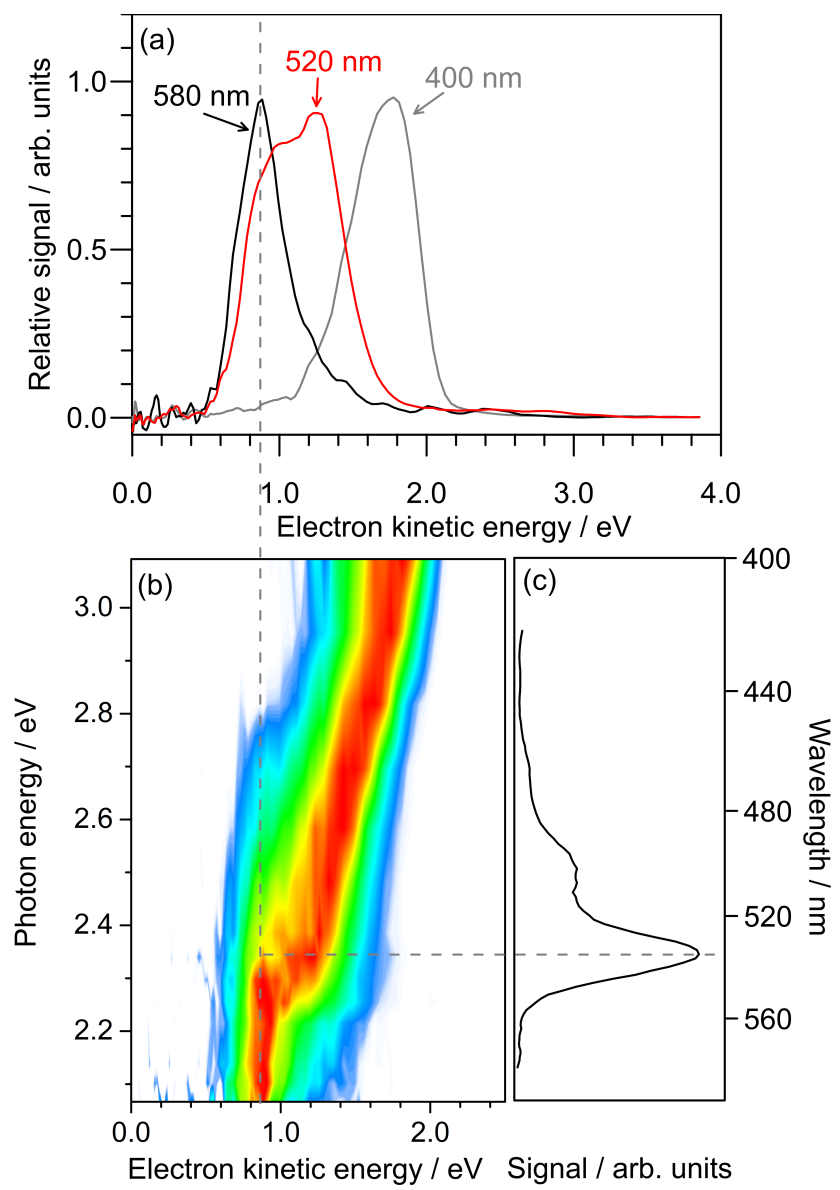

(d)

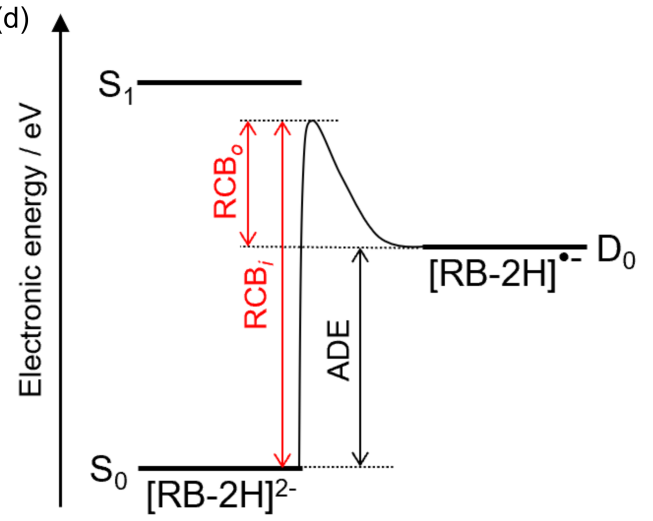

Figure 3: Photoelectron spectroscopy of $[\mathrm{RB}-2 \mathrm{H}]^{2-}$ : (a) example photoelectron spectra at $\lambda=580,520$ and $400 \mathrm{~nm}$ ( $E=2.14,2.38$ and $3.10 \mathrm{eV})$, (b) frequencyresolved photoelectron spectrum, (c) Sep1 photodetachment spectrum reproduced from Figure 2a, (d) schematic potential energy diagram illustrating ADE, $\mathrm{RCB}_{i}$ and $\mathrm{RCB}_{o}$ parameters.

trum in Figure $3 \mathrm{c}$ to indicate the relative photoelectron yield.

According to the photodetachment action spectrum, the photoelectron spectra for $\lambda<430 \mathrm{~nm}$ (photon energy, $E>2.88 \mathrm{eV}$ ) is likely dominated by direct photodetachment because these wavelengths are not resonant with the $\mathrm{S}_{1}\left(\pi \pi^{*}\right) \leftarrow \mathrm{S}_{0}$ transition. These photoelectron spectra provide the vertical detachment energy, VDE $=1.3 \pm 0.1 \mathrm{eV}$, corresponding to the photon energy minus the peak eKE of the photoelectron spectra, and the adiabatic detachment energy, $\mathrm{ADE}=1.1 \pm 0.1 \mathrm{eV}$, corresponding to the photon energy minus the high-eKE edge of the photoelectron spectra. Both values agree with DLPNO-CCSD(T)/def2-SVPD calculations giving $\mathrm{VDE}=1.31 \mathrm{eV}$ and ADE $=1.22 \mathrm{eV}$ (Table 1 ).

For $\lambda>551 \mathrm{~nm}(E<2.25 \mathrm{eV})$, the frequency-resolved spectrum (Figure $3 \mathrm{~b}$ ) is dominated by a photoelectron feature with peak eKE $=0.9 \pm 0.1 \mathrm{eV}, \beta_{2} \approx 0.2 \pm 0.1$ and low-eKE edge at $0.6 \pm 0.1 \mathrm{eV}$. The photodetachment action spectrum, shown in Figure $3 \mathrm{c}$, indicates that these wavelengths are not resonant with the $\mathrm{S}_{1}\left(\pi \pi^{*}\right) \leftarrow \mathrm{S}_{0}$ transition (i.e., longer wavelengths than the absorption band), implying that the photoelectron spectra are dominated by a combination of the high electron kinetic energy tail of direct photodetachment with possibly a small amount of hot-band signal from resonant excitation followed by autodetachment. The low-eKE "cutoff" in these spectra is because of the minimum electron kinetic energy required to overcome the repulsive Coulomb barrier, and provides a direct measure of the outer RCB height, $\mathrm{RCB}_{o}=0.6-0.9 \mathrm{eV}$. The inner-RCB, $\mathrm{RCB}_{i}$, can be calculated from $\mathrm{RCB}_{i}=\mathrm{RCB}_{o}+\mathrm{ADE}$ $=1.7-2.0 \mathrm{eV}$. Ultimately, these data indicate that the $\mathrm{S}_{1}\left(\pi \pi^{*}\right)$ state is situated above the RCB. Note that the photoelectron spectra for $\lambda>551 \mathrm{~nm}(E<2.25 \mathrm{eV})$ are similar because only a fraction of the total photoelectron signal is captured due to the RCB.

For $\lambda \approx 551-430 \mathrm{~nm}(E \approx 2.25-2.88 \mathrm{eV})$ excitation to the $\mathrm{S}_{1}\left(\pi \pi^{*}\right)$ state is possible and there is a clear change in the shape of the photoelectron spectra, presumably because of changes in the Franck-Condon factors. The timescale for autodetachment must be shorter than molecular rotation because the photoelectron angular distribution is anisotropic $\left(\beta_{2} \approx-0.3 \pm 0.1\right)$. Note that the angular anisotropy associated with autodetachment (i.e. resonant electron ejection) differs from the angular anisotropy associated with direct photodetachment (i.e. non-resonant electron ejection). In some cases the non-resonant angular anisotropy can be simulated using point-charge models, ${ }^{62,63}$ however, simulating photoelectron angular distributions from autodetachment is a more challenging task.

The ADE for $[\mathrm{RB}-2 \mathrm{H}]^{2-}$ is nearly twice that for $[\mathrm{F}$ $2 \mathrm{H}]^{2-}(0.5 \pm 0.1 \mathrm{eV}),{ }^{37}$ presumably due to the electron withdrawing halogen atoms in Rose Bengal. Intriguingly, $\mathrm{RCB}_{i}=1.7-2.0 \mathrm{eV}$ for $[\mathrm{RB}-2 \mathrm{H}]^{2-}$ is somewhat lower than that for $[\mathrm{F}-2 \mathrm{H}]^{2-}(2.1-2.2 \mathrm{eV})^{37}$ even though both $[\mathrm{RB}-2 \mathrm{H}]^{2-}$ and $[\mathrm{F}-2 \mathrm{H}]^{2-}$ have similar ground state geometries with equilibrium xanthene-benzoate dihedral angles of $\varphi=90^{\circ}$ to minimize Coulombic repulsion between deprotonation sites. The decreased $\mathrm{RCB}_{i}$ for $[\mathrm{RB}-2 \mathrm{H}]^{2-}$ compared with $[\mathrm{F}-2 \mathrm{H}]^{2-}$ is likely because the negative charge is substantially more delocalized across the iodine and chlorine atoms (supported by Mulliken population analysis - see Supporting Information), lead- 
ing to a decrease in Coulombic repulsion.

We were unable to detect fluorescence signal from [RB-2H $]^{2-}$ when pumping at $533 \mathrm{~nm}$ presumably due to rapid electron detachment. This result is consistent with the absence of fluorescence from $[\mathrm{F}-2 \mathrm{H}]^{2-} .{ }^{20}$

\section{Monoanions - $[\mathrm{RB}-\mathrm{H}]^{-} \&[\mathrm{RB}-2 \mathrm{H}]^{\bullet-}$}

Singly deprotonated Rose Bengal, [RB-H $]^{-}$(which could potentially be generated as phenoxide $\left(\mathrm{O}^{-}\right)$and carboxylate $\left(\mathrm{COO}^{-}\right)$deprotomers $)$, and the radical monoanion, $[\mathrm{RB}-2 \mathrm{H}]^{\bullet-}$, differ in nominal mass by $1 \mathrm{Da}$ (mass-to-charge ratio, $m / z=971.7$ and 972.7). However, it is difficult to isolate these species using lowresolution mass spectrometry techniques; a suitable $m / z$ filter needs resolving power $R>1000$. Fortunately, the monoanions are distinguishable based on their collision cross-section with $\mathrm{N}_{2}$ buffer gas, and their photochemistry can be probed using the IMS-photo-IMS ([RB$\left.\mathrm{H}]^{-}\right)$and photo-IMS-photo-IMS ([RB-2H $\left.]^{\bullet-}\right)$ strategies. ${ }^{42,43}$ Note that (single photon) photodetachment spectroscopy for the $\mathrm{S}_{1}\left(\pi \pi^{*}\right) \leftarrow \mathrm{S}_{0}$ transition is not possible for the monoanions because their ADEs exceed the $\mathrm{S}_{1}\left(\pi \pi^{*}\right)$ band energy (Table 1$)$.

\section{Ion Mobility Spectrometry}

Arrival time distributions (ATDs) for Rose Bengal monoanions electrosprayed from methanol and dry acetonitrile are shown in Figure 4a. For a low RF drive voltage applied to IF1 (IF1 low), the methanol ATD shows a single peak assigned to $[\mathrm{RB}-\mathrm{H}]^{-}$because methanol is a protic solvent, allowing $[\mathrm{RB}-\mathrm{H}]^{-}$to be generated in the electrospray process. Increasing the RF drive voltage (IF1 high) produced a second ATD peak at shorter arrival time. A low RF drive voltage applied to IF1 produces an ATD that reflects the nascent electrosprayed isomer populations. In contrast, a high RF drive voltage applied to IF1 induces energetic collisions between the ions and buffer gas molecules, significantly increasing ion throughput but causing collision-induced electron detachment.

ATDs recorded using dry acetonitrile (non-protic) as the electrospray solvent produced a single ATD peak corresponding to the shorter arrival time peak in the methanol ATD. The shorter arrival time peak is assigned to $[\mathrm{RB}-2 \mathrm{H}]^{\bullet-}$, which can be formed through collision-induced electron detachment of $[\mathrm{RB}-2 \mathrm{H}]^{2-}$. These ATD peak assignments are consistent with the calculated collision cross-sections given in Table 2 , which suggest that the collision cross-section for [RB$2 \mathrm{H}]^{\bullet-}$ is $\approx 5 \AA^{2}$ smaller than for either deprotomer of $[\mathrm{RB}-\mathrm{H}]^{-}$. It is worth noting that the carboxylate deprotomer of $[\mathrm{RB}-\mathrm{H}]^{-}$was calculated to lie $0.26 \mathrm{eV}$ higher in energy than the phenoxide deprotomer (gas-phase calculation at the DLPNO-CCSD(T)/SVPD level of theory), however, the calculated collision cross-sections for each deprotomer are within $1 \AA^{2}$ (Table 2), meaning that the deprotomers are probably not distinguishable
Table 2: Calculated collision cross-sections $\left(\Omega_{c}\right)$ for Rose Bengal monoanion species. $\left(\mathrm{O}^{-}\right)$and $\left(\mathrm{COO}^{-}\right)$indicate phenoxide and carboxylate deprotomers, respectively.

\begin{tabular}{lc}
\hline Species & $\Omega_{c} / \AA^{2}$ \\
\hline$[\mathrm{RB}-\mathrm{H}]^{-}\left(\mathrm{O}^{-}\right)$ & 243 \\
{$[\mathrm{RB}-\mathrm{H}]^{-}\left(\mathrm{COO}^{-}\right)$} & 243 \\
{$[\mathrm{RB}-2 \mathrm{H}]^{\bullet-}$} & 238 \\
\hline
\end{tabular}

in the IMS drift region. Although we are unable to unequivocally say that $[\mathrm{RB}-\mathrm{H}]^{-}$is present as a single deprotomer, we expect the phenoxide deprotomer $\left(\mathrm{O}^{-}\right)$ to be the predominant gas-phase form. This conclusion is consistent with earlier studies on $[\mathrm{F}-\mathrm{H}]^{-} \cdot{ }^{20,22-24,26}$

In another measurement, we irradiated the electrosprayed ions from dry acetonitrile (IF1 low) with light from a $532 \mathrm{~nm}$ YAG laser immediately after the ions were injected into the drift region. ${ }^{42}$ This arrangement produced $[\mathrm{RB}-2 \mathrm{H}]^{\bullet-}$ through photodetachment; the photodetachment action spectra for $[\mathrm{RB}-2 \mathrm{H}]^{2-}$ in Figure $2 \mathrm{a}$ indicates that the radical is efficiently produced

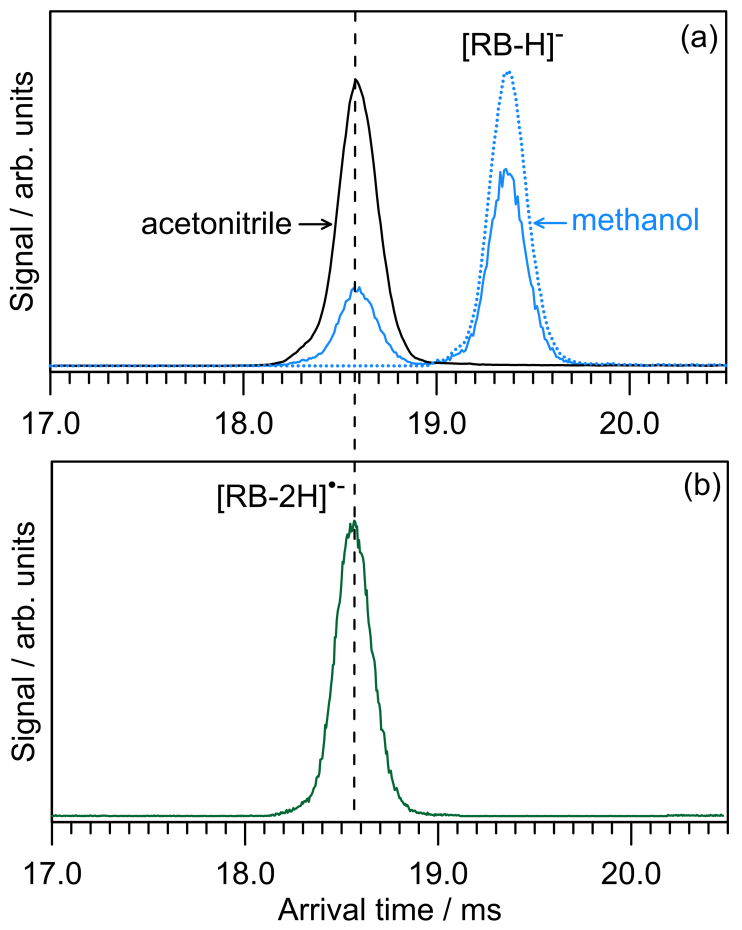

Figure 4: Arrival time distributions (ATDs) for monoanions in pure $\mathrm{N}_{2}$ buffer gas: (a) electrospray from methanol (IF1 low - blue dashed, IF1 high - blue solid) and dry acetonitrile (IF1 high - black solid) solvents, (b) electrospray from dry acetonitrile solvent with IF1 low and irradiation of the ions immediately after they are injected into the drift region with a $532 \mathrm{~nm}$ pulse of light to photogenerate $[\mathrm{RB}-2 \mathrm{H}]^{\bullet-}\left([\mathrm{RB}-2 \mathrm{H}]^{2-} \stackrel{h \nu}{\longrightarrow}[\mathrm{RB}-\right.$ $\left.2 \mathrm{H}]^{\bullet-}+e^{-}\right)$. Note, the quadrupole mass filter was set to transmit ions in the $m / z=965-981$ window. 

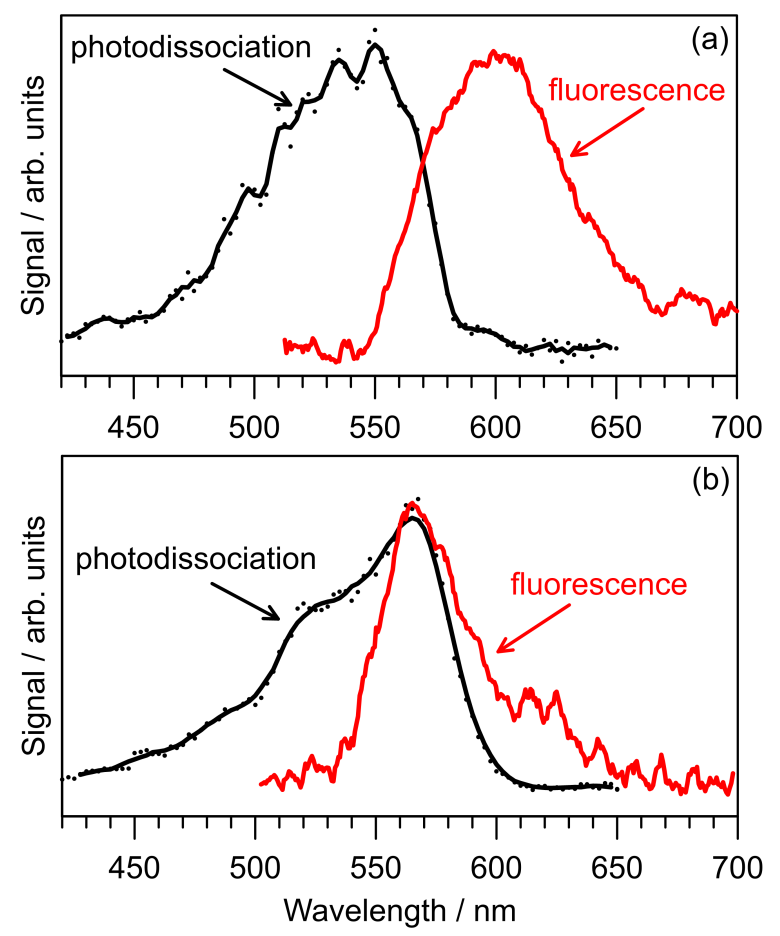

Figure 5: Action spectra for Rose Bengal monoanions: (a) photodissociation spectrum (black) and dispersed fluorescence spectrum (red, pumped at $533 \mathrm{~nm}$ ) for [RB$\mathrm{H}]^{-}$, (b) photodissociation spectrum (black) and dispersed fluorescence spectrum (red, pumped at $533 \mathrm{~nm}$ ) spectra for $[\mathrm{RB}-2 \mathrm{H}]^{\bullet-}$. The fluorescence spectra are 9point moving averages $(0.7 \mathrm{~nm}$ width bins).

at $532 \mathrm{~nm}$. The photogenerated ATD peak for [RB$2 \mathrm{H}]^{\bullet-}$ (Figure $4 \mathrm{~b}$ ) has an arrival time within $0.02 \mathrm{~ms}$ of the shorter arrival time ATD peak, supporting the above ATD peak assignments.

\section{[RB-H $]^{-}$action spectroscopy}

Photodissociation and dispersed fluorescence action spectra for $[\mathrm{RB}-\mathrm{H}]^{-}$are shown in Figure 5a. The photodissociation spectrum was recorded using the IMSphoto-IMS strategy and multi-photon absorption conditions (see Supporting Information). The spectrum has maximum response at $540 \pm 10 \mathrm{~nm}$, which is red shifted compared to the $[\mathrm{F}-\mathrm{H}]^{-}$spectrum $(533 \pm 5 \mathrm{~nm})$ reported by Kjær et al. ${ }^{23,24}$ The spectral profile is broader than the photodetachment spectra for $[\mathrm{RB}-2 \mathrm{H}]^{2-}$ (Figure 2a) and has no clear vibronic structure. Calculated vertical excitation wavelengths for $[\mathrm{RB}-\mathrm{H}]^{-}$(Table 1 ) with the DFT methods (454 and $437 \mathrm{~nm}$ ) are again in poor agreement with experiment. The df-CC2 $(508 \mathrm{~nm})$ and df-ADC $(2)(578 \mathrm{~nm})$ vertical excitation wavelengths are in better agreement with experiment. A FCHT simulation of the absorption spectrum was not performed for this species due to the shallow potential energy surfaces for xanthene-benzoate torsion and calculation convergence difficulties.

Weak fluorescence was observed from $[\mathrm{RB}-\mathrm{H}]^{-}$(elec-
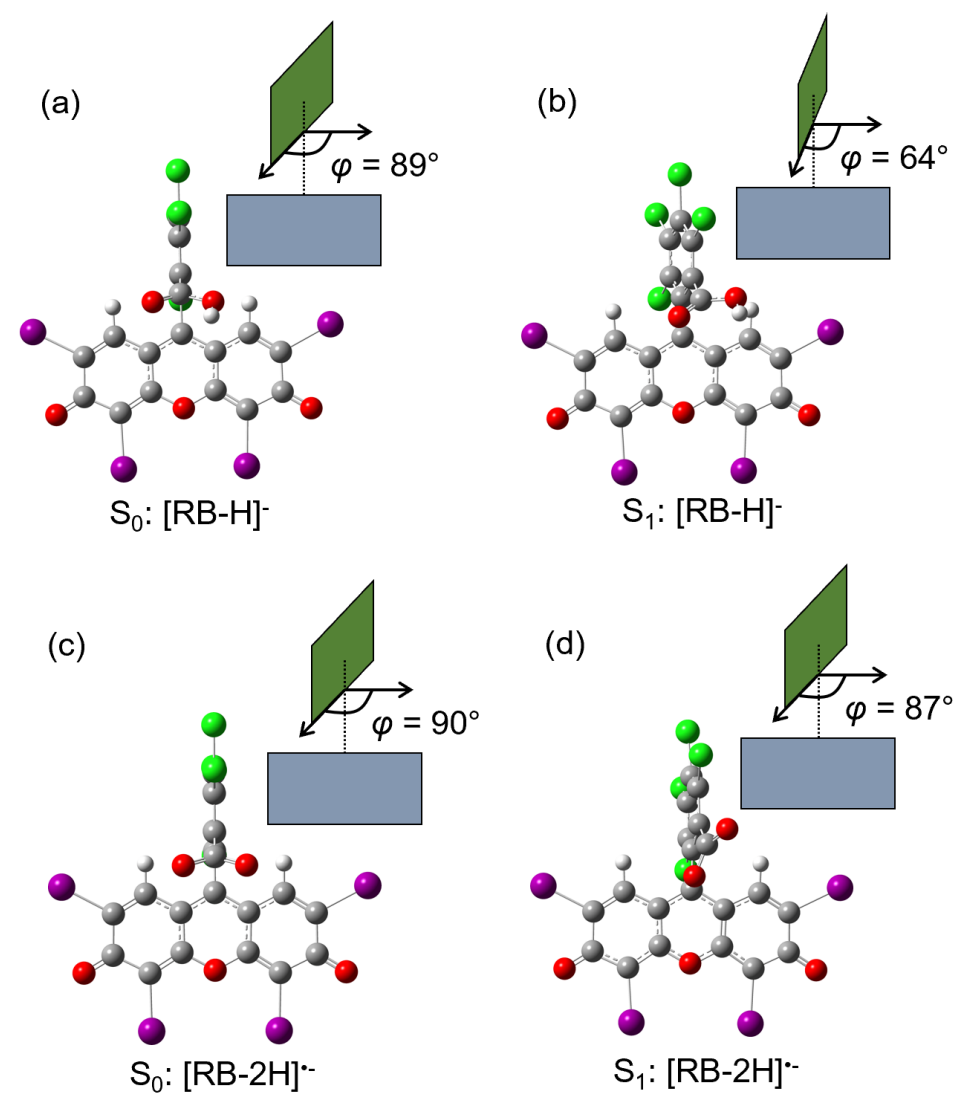

Figure 6: Equilibrium geometries calculated at the $\omega$ B97X-D/def2-SVPD level of theory for phenoxide deprotomers of: (a) $[\mathrm{RB}-\mathrm{H}]^{-}$in the $\mathrm{S}_{0}$ state, (b) $[\mathrm{RB}-\mathrm{H}]^{-}$ in the $\mathrm{S}_{1}\left(\pi \pi^{*}\right)$ state, (c) [RB-2H] $]^{\bullet-}$ in the $\mathrm{S}_{0}$ state, (d) $[\mathrm{RB}-2 \mathrm{H}]^{\bullet-}$ in the $\mathrm{S}_{1}\left(\pi \pi^{*}\right)$ state. Geometric parameter $\varphi$ is the xanthene-benzoate dihedral angle. Note the torsion of the carboxylate group in both $\mathrm{S}_{1}$ geometries.

trosprayed from methanol) when pumped at $533 \mathrm{~nm}$, with the dispersed fluorescence spectrum (Figure 5a) having a peak at $600 \pm 10 \mathrm{~nm}$ and broad spectral profile. Assuming that the peak of the photodissociation action spectrum is the same as the absorption spectrum, gasphase $[\mathrm{RB}-\mathrm{H}]^{-}$has a Stokes shift of $1,700 \pm 200 \mathrm{~cm}^{-1}$, which is comparable with the Stokes shift for gas-phase $[\mathrm{F}-\mathrm{H}]^{-}\left(\approx 2,000 \mathrm{~cm}^{-1}\right) .^{23,24}$

The large Stokes shift is explicable by considering the geometries of $[\mathrm{RB}-\mathrm{H}]^{-}$in the ground and fluorescing states (Figure 6). $[\mathrm{RB}-\mathrm{H}]^{-}$in the ground electronic state has a calculated equilibrium geometry with the xanthene-benzoate dihedral angle of $\varphi=89^{\circ}$. In contrast, the optimized geometry of the $\mathrm{S}_{1}\left(\pi \pi^{*}\right)$ state has $\varphi=64^{\circ}$. A similar situation pertains for $[\mathrm{F}-\mathrm{H}]^{-} .{ }^{24,26}$ For $[\mathrm{RB}-\mathrm{H}]^{-}$, the vertical fluorescence wavelength was calculated as $597 \mathrm{~nm}$ (df-CC2), corresponding to Stokes shift of $\approx 2,900 \mathrm{~cm}^{-1}$, roughly 1.7 -fold larger than the experimental value. 


\section{[RB-2H $]^{\bullet-}$ action spectroscopy}

Photodissociation and dispersed fluorescence action spectra for $[\mathrm{RB}-2 \mathrm{H}]^{\bullet-}$ are shown in Figure 5b. The photodissociation spectrum was recorded using the photoIMS-photo-IMS strategy, which involved photogenerating $[\mathrm{RB}-2 \mathrm{H}]^{\bullet-}$ through photodetachment of $[\mathrm{RB}-2 \mathrm{H}]^{2-}$ using a pulse of $532 \mathrm{~nm}$ light at the start of the drift region - see Supporting Information for the photodissociation spectrum for $[\mathrm{RB}-2 \mathrm{H}]^{\bullet-}$ generated through collision-induced electron detachment of $[\mathrm{RB}-2 \mathrm{H}]^{\bullet-}$. The photodissociation spectrum has maximum response at $563 \pm 5 \mathrm{~nm}$, which is red shifted by $\approx 18 \mathrm{~nm}$ compared to the $[\mathrm{RB}-\mathrm{H}]^{-}$action spectrum (Figure $5 \mathrm{a}$ ). The spectrum has a weak vibronic shoulder at $\approx 520 \mathrm{~nm}$.

The dispersed fluorescence action spectrum for [RB$2 \mathrm{H}]^{\bullet-}$ (Figure 5b) was recorded by exposing trapped [RB-2H $]^{2-}$ to light at sufficient intensity to promote sequential photon absorption. Specifically, no fluorescence was observed from trapped dianions at low laser power $(<1 \mathrm{~mW})$ due to rapid electron detachment, although fluorescence high laser powers $(>10 \mathrm{~mW})$ led to fluorescence signal. This is because absorption of a first photon forms $[\mathrm{RB}-2 \mathrm{H}]^{\bullet-}$ through photodetachment, which is then electronically excited through absorption of a second photon. The dispersed fluorescence spectrum has a maximum response near the maximum of the photodissociation spectrum at $563 \pm 5 \mathrm{~nm}$. Note that some $[\mathrm{RB}-\mathrm{H}]^{-}$formed from electrospray ionization were probably stored together with the dianions and contributed weak fluorescence signal. However, the $[\mathrm{RB}-\mathrm{H}]^{-}$fluorescence signal is much lower than that from the radical species. This was evidenced by the weak fluorescence signal if only $[\mathrm{RB}-\mathrm{H}]^{-}$was stored in the trap.

The lack of an appreciable Stokes shift from the [RB$2 \mathrm{H}]^{\bullet-}$ action spectra is consistent with the calculated geometries for the $\mathrm{S}_{0}$ and $\mathrm{S}_{1}\left(\pi \pi^{*}\right)$ states shown in Figure 6. In this case, $S_{0}$ has $\varphi=90^{\circ}$ and $S_{1}\left(\pi \pi^{*}\right)$ has $\varphi=87^{\circ}$. The calculated vertical fluorescence wavelength at $524 \mathrm{~nm}$ (LC- $\omega \mathrm{HPBE} /$ def2-SVPD level of theory) is close to the calculated vertical excitation wavelength $(521 \mathrm{~nm})$.

\section{Conclusions}

This work demonstrates that Rose Bengal is readily produced as the deprotonated monoanion (from a protic solvent) and doubly deprotonated dianion using electrospray ionization. The doubly deprotonated radical monoanion can also be produced through collisioninduced electron detachment in the ion source, or through exposure of the gas-phase dianion to green light. All three target anions strongly absorb light over the yellow to blue region of the visible spectrum, but have distinct action spectra. The deprotonated monoanion and doubly deprotonated radical monoanion show weak fluorescence in the gas phase because the absorption band lies well below the electron detach- ment threshold and presumably because of a long excited state lifetime. A large Stokes shift of $\approx 1,700 \mathrm{~cm}^{-1}$ for the deprotonated monoanion is consistent with torsion of the xanthene-benzoate dihedral angle to reach the fluorescing state. In contrast, the doubly deprotonated radical monoanion shows no appreciable Stokes shift, consistent with little change in geometry to reach the fluorescing state.

This work complements other gas-phase luminescence studies on ionic dyes, including fluorescein and rhodamines, demonstrating the capacity for xanthenebased dyes to fluoresce in the gas phase. This capacity allows them to be incorporated into biomolecules and potentially inform on gas-phase conformation. For example, fluorescence spectroscopy strategies applied to large biomolecules, e.g. using FRET, ${ }^{9}$ can produce fluorescence signal following the absorption of a single photon which embodies information on the gas-phase structure. ${ }^{8,64,65}$ In contrast, photodissociation studies on large biomolecules such as polypeptides or small proteins incorporating dye molecules usually require absorption of several photons on experimental time scales (e.g. microseconds) to observe a dissociation response.

A significant outcome from this work was use of the photo-IMS-photo-IMS strategy to photogenerate, isolate, and probe ions (e.g. radical anions) that are conventionally difficult to generate in the gas phase. We note that an earlier study using an IMS-photo-IMS approach with a related anionic xanthene dye (Eosin Y) demonstrated photogeneration of doubly deprotonated radical anion through photodetachment of the dianion with $488 \mathrm{~nm}$ light, ${ }^{66}$ although the study was not extended to measure an action spectrum. The photoIMS-photo-IMS methodology used in this work could be applied to other systems for which mass spectrometry separation based on mass-to-charge ratio alone is not sufficient, including transient intermediates in multi-step photoreactions. In the context of using dyes as fluorescent probes for biomolecular structure, the strategy of studying target molecules tagged with dye molecules using both multistage IMS ( + mass-to-charge filtering) and fluorescence spectroscopy should provide a powerful approach for investigating gas-phase structure. For example, ion mobility coupled with electronic spectroscopy can be used to ascertain molecular shapes and shape-specific action spectra for a target molecule, while fluorescence spectroscopy provides the Stokes shift (which is sensitive to local environment of the fluorophore) and can inform on the proximity of coupled dye molecules/chromophores.

\section{Supporting Information Available}

Photodetachment light fluence dependence in Sep1 measurements. Calculated vibrational frequencies for [RB$2 \mathrm{H}]^{2-}$. Ion mobility spectrometry photoaction ATDs and further action spectra. Spectroscopic properties of $[\mathrm{RB}-2 \mathrm{H}]^{2-}$ measured in different solvents. Charge dis- 
tributions for $[\mathrm{F}-2 \mathrm{H}]^{2-}$ and $[\mathrm{RB}-2 \mathrm{H}]^{2-}$ optimized geometries.

\section{Acknowledgements}

Funding was provided by the Swedish Foundation for International Cooperation in Research and Higher Education (STINT, grant number PT2017-7328 to MHS and JNB), a start-up grant at University of East Anglia (to JNB), Australian Research Council's Discovery Project funding scheme (DP150101427 and DP160100474 to EJB), the Independent Research Fund Denmark | Natural Sciences (9040-00041B to SBN), and the European Research Council's Starting Grant scheme (306536 to JRRV). Electronic structure calculations were carried out on the High Performance Computing Cluster supported by the Research and Specialist Computing Support service at the University of East Anglia.

\section{Author Contributions}

Photodetachment experiments using Sep1 and dispersed fluorescence experiments using LUNA were performed by JNB, MHS and CK in the laboratory of SBN. Photoelectron spectroscopy experiments were performed by JNB and SD in the laboratory of JRRV. Ion mobility experiments were performed by JNB in the laboratory of EJB. Electronic structure calculations were performed by JNB. The manuscript was prepared by JNB with contributions from all authors.

\section{Conflicts of Interest}

There are no conflicts of interest to declare.

\section{References}

(1) Neckers, D. C.; Valdes-Aguilera, O. M. In Advances in Photochemistry; Volman, D. H., Hammond, G. S., Neckers, D. C., Eds.; John Wiley \& Sons, Inc., 1993; Vol. 18; Chapter Photochemistry of the Xanthene Dyes, pp 315-394.

(2) Sabnis, R. W. Handbook of Fluorescent Dyes and Probes; Wiley-Blackwell, 2015.

(3) Beija, M.; Afonso, C. A. M.; Martinho, J. M. G. Synthesis and Applications of Rhodamine Derivativesas Fluorescent Probes. Chem. Soc. Rev. 2009, 38, 24102433.

(4) Haugland, R. P. The Molecular Probes Handbook - A Guide to Fluorescent Probes and Labeling Technologies, 10th ed.; Thermo Fisher Scientific, 2005.

(5) Richard, G.; Soubrane, G.; Yanuzzi, L. Fluorescein and ICG Angiography: Textbook and Atlas, 2nd ed.; Thieme, 1998.
(6) Mescher, A. L. Junqueira's Basic Histology: Text and Atlas, 15th ed.; McGraw-Hill Education, 2018.

(7) Giepmans, B. N. G.; Adams, S. R.; Ellisman, M. H.; Tsien, R. Y. The Fluorescent Toolbox for Assessing Protein Location and Function. Science 2006, 312, 217-224.

(8) Talbot, F. O.; Rullo, A.; Yao, H.; Jockusch, R. A Fluorescence Resonance Energy Transfer in Gaseous, Mass-Selected Polyproline Peptides. J. Am. Chem. Soc. 2010, 132, 16156-16164.

(9) Czar, M. F.; Zosel, F.; König, I.; Nettels, D.; Wunderlich, B.; Schuler, B.; Zarrine-Afsar, A.; Jockusch, R. A. Gas-Phase FRET Efficiency Measurements To Probe the Conformation of Mass-Selected Proteins. Anal. Chem. 2015, 87, 7559-7565.

(10) Wysocki, L. M.; Lavis, L. D. Advances in the Chemistry of Small Molecule Fluorescent Probes. Curr. Opinion Chem. Biol. 2011, 15, 752-759.

(11) Ueno, T.; Nagano, T. Fluorescent Probes for Sensing and Imaging. Nat. Methods 2011, 8, 642-645.

(12) Chan, J.; Dodani, S. C.; Chang, C. J. Reaction-Based Small-Molecule Fluorescent Probes for Chemoselective Bioimaging. Nat. Chem. 2012, 4, 973-984.

(13) Ha, T.; Tinnefeld, P. Photophysics of Fluorescent Probes for Single-Molecule Biophysics and SuperResolution Imaging. Annu. Rev. Phys. Chem. 2012, 63, 595-617.

(14) Zhu, H.; Fan, J.; Du, J.; Peng, X. Fluorescent Probes for Sensing and Imaging Within Specific Cellular Organelles. Acc. Chem. Res. 2016, 49, 2115-2126.

(15) Xu, W.; Zeng, Z.; Jiang, J.-H.; Chang, Y.-T.; Yuan, L. Discerning the Chemistry in Individual Organelles with Small-Molecule Fluorescent Probes. Angew. Chem. Int. Ed. 2016, 55, 13658-13699.

(16) Choi, C. M.; Simon, A.-L.; Chirot, F.; Kulesza, A.; Knight, G.; Daly, S.; MacAleese, L.; Antoine, R.; Dugourd, P. Charge, Color, and Conformation: Spectroscopy on Isomer-Selected Peptide Ions. J. Phys. Chem. B 2016, 120, 709-714.

(17) de Vries, M. S.; Hobza, P. Gas-Phase Spectroscopy of Biomolecular Building Blocks. Ann. Rev. Phys. Chem. 2007, 58, 585-612.

(18) Nielsen, S. B., Wyer, J. A., Eds. Photophysics of Ionic Biochromophores; Springer, 2013.

(19) Daly, S.; Kulesza, A.; Knight, G.; MacAleese, L.; Antoine, R.; Dugourd, P. The Gas-Phase Photophysics of Eosin Y and its Maleimide Conjugate. J. Phys. Chem. A 2016, 120, 3484-3490.

(20) McQueen, P. D.; Sagoo, S.; Yao, H.; Jockusch, R. A. On the Intrinsic Photophysics of Fluorescein. Angew. Chem. Int. Ed. 2010, 49, 9193-9196. 
(21) Forbes, M. W.; Jockusch, R. A. Gas-Phase Fluorescence Excitation and Emission Spectroscopy of Three Xanthene Dyes (Rhodamine 575, Rhodamine 590 and Rhodamine 6G) in a Quadrupole Ion Trap Mass Spectrometer. J. Am. Soc. Mass Spectrom. 2011, 22, 93109.

(22) Yao, H.; Jockusch, R. A. Fluorescence and Electronic Action Spectroscopy of Mass-Selected GasPhase Fluorescein, 2', $7^{\prime}$-Dichlorofluorescein, and $2^{\prime}, 7^{\prime}$ Difluorofluorescein Ions. J. Phys. Chem. A 2013, 117, 1351-1359.

(23) Kjær, C.; Nielsen, S. B.; Stockett, M. H. Sibling Rivalry: Intrinsic Luminescence From Two Xanthene Dye Monoanions, Resorufin and Fluorescein, Provides Evidence For Excited-State Proton Transfer in the Latter. Phys. Chem. Chem. Phys. 2017, 22, 9210-9215.

(24) Kjær, C.; Hannson, R. F.; Hedberg, C.; Jensen, F.; Jensen, H. K.; Nielsen, S. B. Gas-phase Action and Fluorescence Spectroscopy of Mass-Selected Fluorescein Monoanions and Two Derivatives. Phys. Chem. Chem. Phys. 2020, 22, 9210-9215.

(25) Horke, D. A.; Chatterley, A. S.; Verlet, J. R. R. Effect of Internal Energy on the Repulsive Coulomb Barrier of Polyanions. Phys. Rev. Lett. 2012, 108, 083003.

(26) Horke, D. A.; Chatterley, A. S.; Bull, J. N.; Verlet, J. R. R. Time-Resolved Photodetachment Anisotropy: Gas-Phase Rotational and Vibrational Dynamics of the Fluorescein Anion. J. Phys. Chem. Lett. 2014, 6, 189-194.

(27) Adamson, B. D.; Coughlan, N. J. A.; Continetti, R. E.; Bieske, E. J. Changing the Shape of Molecular Ions: Photoisomerization Action Spectroscopy in the Gas Phase. Phys. Chem. Chem. Phys. 2013, 15, 9540-9548.

(28) Adamson, B. D.; Coughlan, N. J. A.; da Silva, G.; Bieske, E. J. Photoisomerization Action Spectroscopy of the Carbocyanine Dye DTC ${ }^{+}$in the Gas Phase. $J$. Phys. Chem. A 2013, 117, 13319-13325.

(29) Bull, J. N.; Coughlan, N. J. A.; Bieske, E. J. ProtomerSpecific Photochemistry Investigated Using Ion Mobility Mass Spectrometry. J. Phys. Chem. A 2017, 121, 6021-6027.

(30) Wang, X.-B.; Wang, L.-S. Observation of Negative Electron-Binding Energy in a Molecule. Nature 1999, 400, 245-248.

(31) Dau, P. D.; Liu, H.-T.; Yang, J.-P.; Winghart, M.-O.; Wolf, T. J. A.; Unterreiner, A.-N.; Weis, P.; Miao, Y.R.; Ning, C.-G.; Kappes, M. M. et al. Resonant Tunneling Through the Repulsive Coulomb Barrier of a Quadruply Charged Molecular Anion. Phys. Rev. A 2012, 85 .

(32) Støchkel, K.; Milne, B. F.; Nielsen, S. B. Absorption Spectrum of the Firefly Luciferin Anion Isolated in Vacuo. J. Phys. Chem. A 2011, 115, 2155-2159.
(33) Wyer, J. A.; Brøndsted Nielsen, S. Absorption by Isolated Ferric Heme Nitrosyl Cations In Vacuo. Angew. Chem. Int. Ed. 2012, 51, 10256-10260.

(34) West, C. W.; Bull, J. N.; Antonkov, E.; Verlet, J. R. R. Anion Resonances of para-Benzoquinone Probed by Frequency-Resolved Photoelectron Imaging. J. Phys. Chem. A 2014, 118, 11346-11354.

(35) Bull, J. N.; West, C. W.; Verlet, J. R. R. On the Formation of Anions: Frequency-, Angle-, and TimeResolved Photoelectron Imaging of the Menadione Radical Anion. Chem. Sci. 2015, 6, 1578-1589.

(36) Roberts, G. M.; Lecointre, J.; Horke, D. A.; Verlet, J. R. R. Spectroscopy and Dynamics of the 7,7,8,8-tetracyanoquinodimethane Radical Anion. Phys. Chem. Chem. Phys. 2010, 12, 6226-6232.

(37) Horke, D. A.; Roberts, G. M.; Lecointre, J.; Verlet, J. R. R. Velocity-Map Imaging at Low Extraction Fields. Rev. Sci. Instrum. 2012, 83, 063101.

(38) Roberts, G. M.; Nixon, J. L.; Lecointre, J.; Wrede, E.; Verlet, J. R. R. Toward Real-Time Charged-Particle Image Reconstruction Using Polar Onion-Peeling. Rev. Sci. Instrum. 2009, 80, 053104.

(39) Zare, R. N. Photoejection Dynamics. Mol. Photochem. 1972, 4, 1-37.

(40) Adamson, B. D.; Coughlan, N. J. A.; Markworth, P. B.; Continetti, R. E.; Bieske, E. J. An Ion Mobility Mass Spectrometer for Investigating Photoisomerization and Photodissociation of Molecular Ions. Rev. Sci. Instr. 2014, 85, 123109.

(41) Eiceman, G. A.; Karpas, Z.; Hill, H. H. Ion Mobility Spectrometry, 3rd ed.; CRC Press, 2013.

(42) Bull, J. N.; Scholz, M. S.; Carrascosa, E.; da Silva, G.; Bieske, E. J. Double Molecular Photoswitch Driven by Light and Collisions. Phys. Rev. Lett. 2018, 120, 223002 .

(43) Bull, J. N.; Scholz, M. S.; Carrascosa, E.; Bieske, E. J. From $E$ to $Z$ and Back Again: Reversible Photoisomerisation of an Isolated Charge-Tagged Azobenzene. Phys. Chem. Chem. Phys. 2018, 20, 509-513.

(44) Stockett, M. H.; Houmøller, J.; Støchkel, K.; Svendsen, A.; Nielsen, S. B. A Cylindrical Quadrupole Ion Trap in Combination with an Electrospray Ion Source for Gas-Phase Luminescence and Absorption Spectroscopy. Rev. Sci. Instrum. 2016, 87, 053103.

(45) Stockett, M. H.; Houmøller, J.; Nielsen, S. B. Nile Blue Shows Its True Colors in Gas-Phase Absorption and Luminescence Ion Spectroscopy. J. Chem. Phys. 2016, 145, 104303

(46) Frisch, M. J.; Trucks, G. W.; Schlegel, H. B.; Scuseria, G. E.; Robb, M. A.; Cheeseman, J. R.; Scalmani, G.; Barone, V.; Mennucci, B.; Petersson, G. A. et al. Gaussian 16 Revision B.01. Gaussian Inc. Wallingford CT 2016. 
(47) Neese, F. The ORCA Program System. WIRES Comp. Mol. Sci. 2012, 2, 73-78.

(48) Kállay, M.; Rolik, Z.; Csontos, J.; Nagy, P.; Samu, G.; Mester, D.; Ladjánszki, I.; Szegedy, L.; Ladóczki, B.; Petrov, K. et al. MRCC, A Quantum Chemical Program Suite. www.mrcc.hu.

(49) Chai, J.-D.; Head-Gordon, M. Long-Range Corrected Hybrid Density Functionals with Damped Atom-Atom Dispersion Corrections. Phys. Chem. Chem. Phys. 2008, 10, 6615-6620.

(50) Vydrov, O. A.; Heyd, J.; Krukau, A. V.; Scuseria, G. E. Importance of Short-Range Versus LongRange Hartree-Fock Exchange for the Performance of Hybrid Density Functionals. J. Chem. Phys. 2006, 125, 074106.

(51) Weigend, F.; Ahlrichs, R. Balanced Basis Sets of Split Valence, Triple Zeta Valence and Quadruple Zeta Valence Quality for H to Rn: Design and Assessment of Accuracy. Phys. Chem. Chem. Phys. 2005, 7, 32973305 .

(52) Rappoport, D.; Furche, F. Property-Optimized Gaussian Basis Sets for Molecular Response Calculations. J. Chem. Phys. 2010, 133, 134105.

(53) Peterson, K. A.; Figgen, D.; Goll, E.; Stoll, H.; Dolg, M. Systematically Convergent Basis Sets With Relativistic Pseudopotentials. II. Small-Core Pseudopotentials and Correlation Consistent Basis Sets for the Post-d Group 16-18 Elements. J. Chem. Phys. 2003, 119, 11113-11123.

(54) Santoro, F.; Lami, A.; Improta, R.; Bloino, J.; Barone, V. Effective Method for the Computation of Optical Spectra of Large Molecules at Finite Temperature Including the Duschinsky and Herzberg-Teller Effect: The Qx Band of Porphyrin as a Case Study. J. Chem. Phys. 2008, 128, 224311.

(55) Hättig, C.; Weigend, F. CC2 Excitation Energy Calculations on Large Molecules Using the Resolution of the Identity Approximation. J. Chem. Phys. 2000, 113, $5154-5161$

(56) Schirmer, J.; Trofimov, A. B. Intermediate State Representation Approach to Physical Properties of Electronically Excited Molecules. J. Chem. Phys. 2004, 120, 11449-11464.

(57) Dutta, A. K.; Saitow, M.; Demoulin, B.; Neese, F.; Izsák, R. A Domain-Based Local Pair Natural Orbital Implementation of the Equation of Motion Coupled Cluster Method for Electron Attached States. J. Chem. Phys. 2019, 150, 164123.

(58) Campuzano, I.; Bush, M. F.; Robinson, C. V.; Beaumont, C.; Richardson, K.; Kim, H.; Kim, H. I. Structural Characterization of Drug-Like Compounds by Ion Mobility Mass Spectrometry: Comparison of Theoretical and Experimentally Derived Nitrogen Collision cross sections. Anal. Chem. 2012, 84, 1026-1033.
(59) Mesleh, M. F.; Hunter, J. M.; Shvartsburg, A. A.; Schatz, G. C.; Jarrold, M. F. Structural Information From Ion Mobility Measurements: Effects of the LongRange Potential. J. Phys. Chem. 1996, 100, 1608216086.

(60) Besler, B. H.; Merz, Jr., K. M.; Kollman, P. A. Atomic Charges Derived From Semiempirical Methods. J. Comp. Chem. 1990, 11, 431-439.

(61) Kulesza, A. J.; Titov, E.; Daly, S.; Włodarczyk, R.; Megow, J.; Saalfrank, P.; Choi, C. M.; MacAleese, L.; Antoine, R.; Dugourd, P. Excited States of Xanthene Analogues: Photofragmentation and Calculations by CC2 and Time-Dependent Density Functional Theory. ChemPhys Chem 2016, 17, 3129-3138.

(62) Ning, C.-G.; Dau, P. D.; Wang, L.-S. Guiding Electron Emissions by Excess Negative Charges in Multiply Charged Molecular Anions. Phys. Rev. Lett. 2010, 105.

(63) West, C. W.; Bull, J. N.; Woods, D. A.; Verlet, J. R. R. Photoelectron Imaging as a Probe of the Repulsive Coulomb Barrier in the Photodetachment of Antimony Tartrate Dianions. Chem. Phys. Lett. 2016, $645,138-143$.

(64) Kjær, C.; Zhao, Y.; Stockett, M. H.; Chen, L.; Hansen, K.; Nielsen, S. B. Gas-Phase Förster Resonance Energy Transfer in Mass-Selected Ions With Methylene or Peptide Linkers Between Two Dyes: A Concerted Dance of Charges. Phys. Chem. Chem. Phys. 2020, 22, 11095-11100.

(65) Czar, M. F.; Jockusch, R. A. Sensitive Probes of Protein Structure and Dynamics in Well-Controlled Environments: Combining Mass Spectrometry With Fluorescence Spectroscopy. Curr. Op. Struct. Bio. 2015, $34,123-134$

(66) Simon, A.-L.; Chirot, F.; Choi, C. M.; Clavier, C.; Barbaire, M.; Maurelli, J.; Dagany, X.; MacAleese, L.; Dugourd, P. Tandem Ion Mobility Spectrometry Coupled to Laser Excitation. Rev. Sci. Instrum. 2015, 86, 094101. 
Graphical TOC Entry

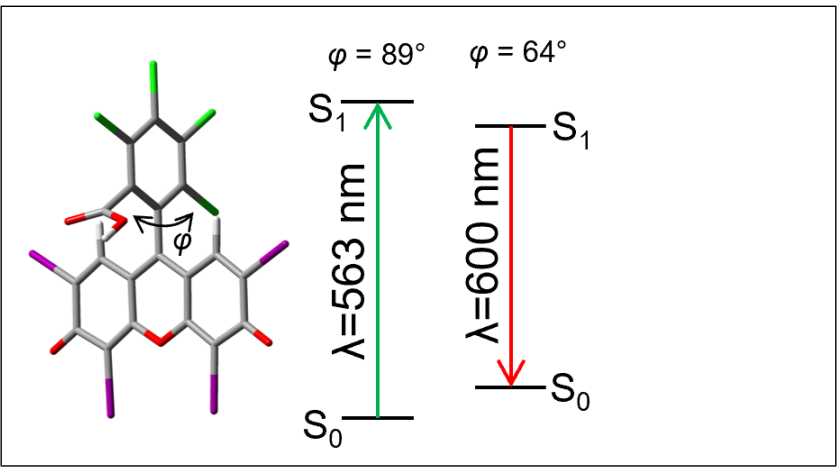

